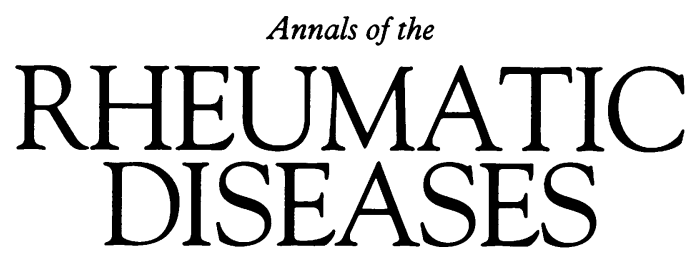

Leader

\title{
Predicting outcome in current clinic attenders: a biased view
}

Case series and cross-sectional surveys of patients attending specialist clinics are important methods both for recognising and characterising new syndromes, and for formulating hypotheses concerning possible risk factors for disease susceptibility and outcome. In such descriptive studies information about disease status and risk factors is collected simultaneously at one point in time in patients with varying disease duration. The purpose of this editorial is to draw attention to a problem which arises when such information is used as a basis for inferring cause and effect.

To illustrate the problem we have used a paper published recently in the Annals ${ }^{1}$ although previous rheumatological reports have many others. ${ }^{2}{ }^{3}$ The paper describes a 1992 survey of current outpatient attenders at a lupus clinic, using retrospective data from case note review. The records of a small number of patients who had died before the study year were also reviewed. The authors concluded that "factors associated with social deprivation may increase disease severity in SLE". The merit of using this example is that the authors appropriately raised left censorship as a possible source of bias in their study and attempted to examine the problem using available data. What is left censorship and how may it have influenced their findings?

The ideal method of studying the link between social deprivation and disease severity in SLE would be to carry out a prospective study of patients who had newly developed the disease (termed an inception cohort). Measures of social deprivation would be recorded at first presentation, and subsequent progress of the disease monitored. The term cohort refers to a group of patients, each selected on a similar basis, who are followed up over time and whose subsequent outcome is recorded. In this context, outcome includes loss to follow up as well as the disease measures under investigation.

The problem facing those carrying out research into chronic diseases such as SLE is that it takes many years to assemble and follow up a prospective cohort. Instead a group of patients is often assembled from current clinic attenders, and is assumed to represent a cohort. Characteristics such as current social class are then linked with current disease status and used to infer cause and effect. This is where left censorship comes in.

Consider five theoretical patients (A-E) (figure) who all presented in 1980. They would have been part of "the inception cohort of new clinic attenders with SLE in 1980". In 1992, when the present cross-sectional study was performed, only patient A was still attending and "caught" in the cross-sectional survey. Patients B and D had died before the study date. Patients $\mathrm{C}$ and $\mathrm{E}$ had been lost to follow up and were not recruited in the study. None of these latter four members of the 1980 inception cohort would have been identified by a cross-sectional study carried out in 1992. They would have been censored before (to the left of) the study year.

Why should left censorship give rise to bias? Loss to follow up in this example may relate to disease status (patients with nephritis may be more likely to continue to attend than those without) or may relate to socioeconomic factors (which are being investigated as predictors of disease status). This may mean that a cross-sectional sample is not representative of all patients with SLE who attend hospital at some time during the course of their disease. However, this alone will not distort an observed link between nephritis and socioeconomic factors. Such a bias will only arise if the association itself is different in current clinic attenders compared with that in patients lost to follow up. For example, patients of a lower social group may continue attending clinic only if they develop a severe complication such as nephritis, whereas patients of a higher social group may continue to attend irrespective of the severity of their disease. This will lead to an apparent link between low social group and severe disease in the group of patients who continue to attend clinic. This link has arisen because of left censorship and does not represent a true association.

Left censorship bias is a particular problem for studies investigating associations between disease features and variables such as social class, age, or disease duration which may be associated with disease status and, separately, with continuing attendance at clinic. In reality, associations between disease status and such variables may be impossible to interpret from cross-sectional studies.

Hypothetical inception cohort of patients attending a lupus clinic

1980

A
B
D
E

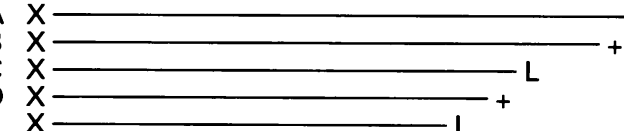

$A-E$ are five patients who from a hypothetical inception cohort assembled in 1980. A cross-sectional study was performed in 1992. Patients B-E were censored before (to the left of) the 1992 study because of death (+) or loss to follow up $(L)$. 
Studies with other aims may be feasible: factors such as HLA or autoantibody status, although they will influence disease outcome, are unlikely separately to influence whether or not patients continue to attend clinic.

The possibility of left censorship bias needs to be considered in cross-sectional studies. ${ }^{3}{ }^{4}$ Results from such studies must be interpreted with caution because the difficulty of obtaining information about the number and characteristics of patients lost to follow up means that it is often difficult to estimate the size of the effect of left censorship bias. Incidence registries would facilitate the task of identifying a true inception cohort for hospital series using retrospective data, ${ }^{5}$ otherwise a time-consuming trawl through the records is required. However, the only certain remedy is to conduct prospective cohort studies, thus ensuring that outcome data are available on all eligible patients independent of their current follow up status.
Correspondence to: Dr Elaine M Hay, ARC Epidemiology Research Unit,

University of Manchester,

Stopford Building,

Oxford Rd,

Manchester M13 9PT,

United Kingdom

1 McAlindon, Giannotta L, Taub N, D'Cruz D, Hughes G. Environmenta factors predicting nephritis in systemic lupus erythematosus. Ann Rheum fistors predicting neph

2 Calin A, Elswood J, Rigg S, Skevington S M. Ankylosing spondylitis-an analytical review of 1500 patients: the changing pattern of disease. $\mathcal{F}$ Rheumatol 1988; 15: 1234-8.

3 Fries J F, Singh G, Bloch D A, Calin A. The natural history of ankylosing spondylitis: is the disease really changing? $f$ Rheumatol 1989; 16: 860-3.

4 Van der Linden S M, Tugwell P. Pitfalls in assessment of prognosis. Weak study designs are liable to bias. $\mathcal{F}$ Rheumatol $1988 ; 15: 725-27$.

5 McCarty D J, Kwoh C K, LaPorte R E. The importance of incidence registries for connective tissue diseases. $\mathcal{F}$ Rheumatol 1992; 19: 1-7. 